\title{
PREDICTING RESPONSE TO CRT IN PATIENTS WITH HF
}

Cardiac resynchronization therapy (CRT) is a well-recognized therapeutic option for patients with moderate-to-severe heart failure (HF), but approximately one third of patients undergoing CRT do not experience clinical improvement. A subanalysis of the PROSPECT trial has found that those who respond best to CRT are more likely to be female, have nonischemic etiology of HF, wider QRS complexes, no history of ventricular tachycardia, and more extensive left ventricular dyssynchrony. "Over time, we have learned that end-stage HF is a complex situation and that not one single parameter is sufficient to predict response to CRT" comments Jeroen Bax, an investigator involved in this subanalysis.

The PROSPECT trial was a large, multicenter study that evaluated whether echocardiographic measures of mechanical ventricular dyssynchrony could be used to predict response to CRT in patients with left ventricular ejection fraction $\leq 35 \%$, NHYA class III or IV HF, and a QRS interval $\geq 130 \mathrm{~ms}$. The first report from the PROSPECT trial caused confusion about the value of assessing left ventricular dyssynchrony for predicting the patient's response to CRT because the sensitivity and specificity of various echocardiographic markers were modest. A subanalysis of the data was thus performed to better characterize the relationship between clinical and echocardiographic measures before and after CRT.

The echocardiographic responses of the patients included in this subanalysis were classified according to reductions in left ventricular end-systolic volume (LVESV) measured at the 6-month follow-up. 'Super responders' achieved a LVESV reduction of at least $30 \%$ from baseline $(n=108)$, 'responders' had a reduction of 15-29\% $(n=53)$, 'nonresponders' did not have a significant reduction in LVESV (that is, $0-14 \% ; n=67$ ), and those classified as 'negative responders' had an increase in LVESV $(n=58)$. Male sex, NYHA class IV status, and history of ventricular tachycardia were all associated with worse echocardiographic response to CRT. In contrast, nonischemic etiology of HF, wider QRS intervals, more left ventricular dyssynchrony, and longer times to peak systolic velocity were associated with better echocardiographic response.

Of the 286 patients assessed, $73 \%$ experienced some beneficial clinical response, as defined by hospitalization-free survival, improvement in NYHA class, or moderate-to-marked improvement in patient global assessment score. Beneficial clinical and echocardiographic responses were observed in $45 \%$ of patients, and this combined positive response was strongly associated with nonischemic etiology of HF. Patients with no clinical or echocardiographic improvement (15\% of the study population) were more likely to have a history of ventricular tachycardia.

"A perfect predicition will never be possible," comments Bax, but he does believe that we will soon be able to classify patients as having low and high likelihood of success of CRT, before they undergo implantation. According to Bax, "there will always be a gray zone-intermediate likelihood of success-[but] that is [the case for] all complex diseases, and certainly holds true for HF."

Bryony M. Mearns

Original article van Bommel, R. J. et al. Characteristics of heart failure patients associated with good and poor response to cardiac resynchronization therapy: a PROSPECT (Predictors of Response to CRT) sub-analysis. Eur. Heart J. 30, 2470-2477 (2009). 